\title{
Predictors of Mortality in Patients with Chronic Heart Failure: Is Hyponatremia a Useful Clinical Biomarker?
}

This article was published in the following Dove Press journal: International Journal of General Medicine

\section{Manal M Alem (1) \\ Department of Pharmacology, College of Clinical Pharmacy, Imam Abdulrahman Bin Faisal University, Dammam, Saudi Arabia}

Background: Chronic heart failure (CHF) is a global health burden. Despite advances in treatment, there remain well-recognised morbidity and mortality. Risk stratification requires the identification and validation of biomarkers, old and new. Hyponatremia has re-emerged as a prognostic marker in CHF patients.

Methods: This is a retrospective cohort study on $241 \mathrm{CHF}$ patients recruited from King Fahd Hospital of the University, Al-Khobar, Saudi Arabia (January 2005-December 2016). Their serum sodium and biochemical parameters were measured at baseline, along with 2-D echocardiographic assessments of left ventricular mass and ejection fraction. The primary endpoint was the association between hyponatremia and all-cause mortality (ACM) after a follow-up period of 24 months.

Results: Mean age of patients was $60.61 \pm 12.63$ (SD) years; $65.1 \%$ were males, and type 2 diabetes mellitus (DM) was present in $71 \%$. Baseline serum sodium was $138.00(136,140)$ (median and interquartile range). Hyponatremia $(<135 \mathrm{meq} / \mathrm{L})$ was present in $14.1 \%$. After follow-up, 46 deaths had occurred. Multivariate Cox-proportional hazard model showed that type 2 DM, New York Heart Association (NYHA) class (III-IV vs I-II), age, and left ventricular mass index (LVMI) were significant and independent predictors of ACM, with HR 3.03 (95\% CI; 1.13, 8.16) ( $\mathrm{P}=0.028)$, HR 2.31 (95\% CI; 1.11, 4.82) ( $\mathrm{P}=0.026)$, HR 1.06 (95\% CI; 1.03, 1.09) $(\mathrm{P}<0.001)$, and HR 1.01 (95\% CI; 1.00, 1.02) $(\mathrm{P}=0.039)$, respectively. Estimated glomerular filtration rate (eGFR) was not a significant predictor. Kaplan-Meier survival analysis was used for the analysis of NYHA class and hyponatremia interactions and showed that hyponatremia had an association with poorer survival in patients with NYHA class III-IV rather than I-II (Log-rank test, $\mathrm{P}=0.0009$ ).

Conclusion: Hyponatremia was a feature in $\mathrm{CHF}$ patients, and ACM was predicted by type 2 DM, NYHA class, age, and LVMI. Hyponatremia impact on survival was in patients with more advanced disease.

Keywords: hyponatremia, electrolyte disturbance, heart failure, left ventricular mass index, ejection fraction, all-cause mortality, Saudi Arabia

\section{Introduction}

Chronic heart failure (CHF) is an established public health problem with significant morbidity and mortality, particularly among patients aged over 65 years. ${ }^{1}$ It has a complex pathogenesis involving lots of genetic and environmental factors. ${ }^{2}$ Despite established diagnostic criteria and up-to-date management guidelines, survival is enormously compromised and falls behind other serious conditions. ${ }^{3}$ Appropriately, the persistent search for biomarkers with an adequate cost/benefit ratio that can
Correspondence: Manal M Alem Department of Pharmacology, College of Clinical Pharmacy, Imam Abdulrahman Bin Faisal University, PO Box 1982, Dammam 3I44I, Saudi Arabia

Email malem@iau.edu.sa 
guide the management plans and predict future outcomes remains a necessity. Older age and disease severity quantified by the New York Heart Association (NYHA) class are important predictors of mortality. ${ }^{4}$ Reduced ejection fraction is an established and strong predictor of cardiovascular outcomes in patients with $\mathrm{CHF}^{4,5}$ Anemia, which is a relatively common pathology, has been found to be an independent prognostic factor for survival in CHF patients, ${ }^{6}$ although the contribution of expanded plasma volume and pseudoanemia complicates the usefulness of serum hemoglobin as a prognostic marker. ${ }^{7}$ More than 3 decades ago, a study reported serum sodium as a powerful predictor of survival in a cohort of 203 patients with severe CHF (defined as ejection fraction $\mathrm{EF}<30 \%$ ) during a follow-up duration of 6-94 months. ${ }^{8}$ This finding was supported by later studies that involved CHF outpatients, ${ }^{9}$ ambulatory patients with heart failure and preserved/ reduced $\mathrm{EF},{ }^{10}$ and heart failure patients with NYHA class III-IV, ${ }^{11}$ and consolidated further by a meta-analysis that included 14,766 patients and reinforced the prognostic value of hyponatremia in CHF patients with reduced or preserved ejection fraction. ${ }^{12}$

Genetic factors, on the other hand, have demonstrated an impact on vulnerability to $\mathrm{CHF}$, on disease progression, and on the response to pharmacological agents. ${ }^{2}$ Therefore, hyponatremia in CHF patients, that is known to be attributed by volume status fluctuation, neurohormonal factors, and diuretics use, ${ }^{13}$ might exhibit different behavior in other patient populations, and this compromises its prognostic value. Therefore, we sought, via this observational study, to assess the prognostic value of hyponatremia in a sample of CHF patients in Saudi Arabia, especially after a recently developed regional registry began to give essential information about this population to be of younger age, with higher rates of diabetes mellitus (DM), and with predominant left ventricular systolic dysfunction. ${ }^{14}$

\section{Methods}

\section{Study Design and Setting}

This is an observational study with a retrospective cohort design to assess the association between hyponatremia and $\mathrm{ACM}$, during a 24-month follow-up period (from the time serum electrolyte measurements were taken), in a sample of CHF patients treated with standard anti-failure drugs. The diagnosis of heart failure was in accordance with the American College of Cardiology/American Heart Association. ${ }^{15}$ Patients were on sodium restriction (defined as $<3$ grams/day) and fluid restriction $(<1.5$ liter/day). The reporting system of this study was in accordance with the Strengthening the Reporting of Observational Studies in Epidemiology (STROBE) statement. ${ }^{16}$

\section{Participants}

Patients were recruited from King Fahd Hospital of the University (KFHU), Al-Khobar, Saudi Arabia- a public health care system affiliated to Imam Abdulrahman Bin Faisal University. The hospital uses QuadraMed, a computerized database that identifies each patient's medical records by a unique number (unit numbering) which is preserved even after the patient's death.

\section{Ethical Approval}

The protocol was approved by the Institutional Review Board (IRB-2020-306-Pharm), Deanship of Scientific Research, Imam Abdulrahman Bin Faisal University, Dammam, Saudi Arabia. The study was conducted in accordance with the Declaration of Helsinki (2013), the ICH Harmonized Tripartite Good Clinical Practice Guidelines, and the laws of Saudi Arabia. Verbal informed consent was obtained from the patients included in the study or their next of kin by telephone conversation. The consent process was approved by the Institutional Review Board, Imam Abdulrahman Bin Faisal University. In addition, electronic medical records were reviewed for study endpoints.

\section{Inclusion and Exclusion Criteria}

The following inclusion and exclusion criteria were applied at the time of baseline data collection or study entry.

\section{Inclusion Criteria}

- Age $>18$ years

- Chronic heart failure with New York Heart Association NYHA functional class I-IV, due to any etiology, and maintained on anti-failure medication for a minimum of 3-month duration.

- Active medical records, and updated contact information.

\section{Exclusion Criteria}

- Acute heart failure

- Serum $\mathrm{Hb}<9 \mathrm{gr} / \mathrm{dl}$ and hematocrit $<30 \%$ due to acquired anemia (with documented serum ferritin, transferrin saturation, or macrocytosis on peripheral 
blood smear), or due to inherited hemoglobinopathy such as; sickle cell disease, and thalassemia (with documented hemoglobin electrophoresis)

- Severe reduction of kidney function (eGFR $<30 \mathrm{~mL} /$ $\mathrm{min} / 1.73 \mathrm{~m}^{2}$ and serum creatinine $>2.5 \mathrm{mg} / \mathrm{dl}$ )

- Chronic inflammatory/autoimmune diseases

- Malignancy

\section{Search Strategy}

The search for eligible patients was done via QuadraMed. Search term via the diagnosis of "Heart/cardiac failure", was used for outpatient and inpatient records from Jan 1st, 2005 till Dec 31st, 2016. This search yielded 876 patients. For the reasons mentioned above, $635 \mathrm{CHF}$ patients were excluded leaving 241 patients for the final analysis (Figure 1).

\section{Variables}

The following data were collected: demographic information (age, sex, height, weight, body mass index (BMI), nationality), NYHA functional class, etiology of heart failure, and comorbidities (systemic hypertension, diabetes mellitus (DM), dyslipidemia, arrhythmias (including atrial fibrillation (AF)), transient ischemic attacks (TIA), stroke, chronic obstructive pulmonary disease (COPD), bronchial asthma, and chronic liver disease). Patients' cardiac medications were extracted (on matching dates), which included angiotensin-converting enzyme inhibitors (ACEI), angiotensin receptor blockers (ARB), beta-blockers, calcium channel blockers (CCBs), antiplatelet agents, anticoagulants, thiazide/loop diuretics, aldosterone receptor antagonists, digoxin, nitrates, and statins. Anti-diabetic medications were also recorded (insulin preparations, sulphonylureas, biguanides, thiazolidinediones,

Patients search in King Fahd Hospital of the University administrative system with search terms "Heart failure", or "Cardiac failure" from Jan $1^{\text {st }}, 2005$ till Dec $31^{\text {st }} 2016$ revealed 876 patients

635 CHF patients were excluded for the following reasons;

139 patients did not meet age criterion 111 patients had an acute heart failure 15 patients had sickle cell disease 39 patients had acquired nutritional anemia 90 patients with chronic kidney disease stage $\geq 4$ 13 patients with chronic inflammatory/ autoimmune conditions

23 patients had cancer

205 patients with inactive medical records or missing contact information
241 CHF patients underwent primary screening for inclusion/ exclusion criteria and their data were extracted and tabulated

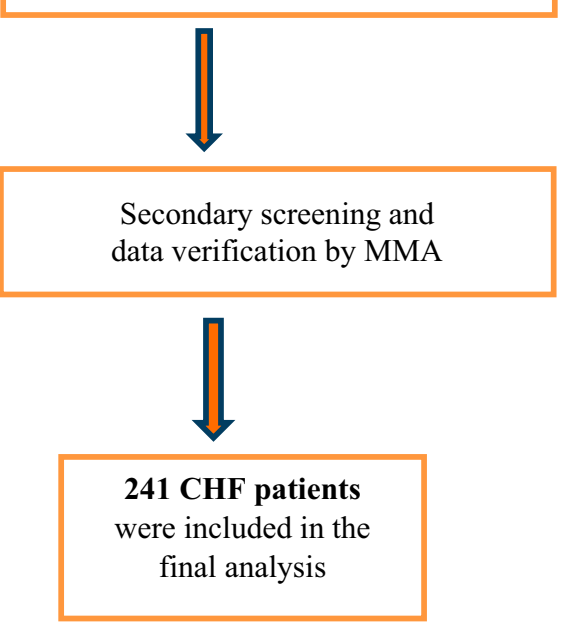

Figure I Study flow chart. 
and dipeptidyl peptidase-4 inhibitors). Hemodynamic and echocardiographic parameters were also extracted.

\section{Measurement of Serum Electrolytes, Biochemical, and Hematological Parameters}

Serum electrolytes, serum creatinine, and blood urea nitrogen were measured using Dimension EXL with LM integrated chemistry system (Siemens Ltd Saudi ArabiaHealthcare, Riyadh, Saudi Arabia). Glomerular filtration rate (GFR) was calculated using the Modification of Diet in Renal Disease equation. ${ }^{17}$ Serum glycated hemoglobin (HbA1C) was measured using Tosoh automated glycohemoglobin analyzer (G8)(Griesheim, Germany). Serum uric acid was measured with Dimension EXL 200 integrated chemistry system (Siemens Ltd Saudi Arabia-Healthcare, Riyadh, Saudi Arabia). Complete blood count (CBC) parameters, including white blood cell count (WBCs), red blood cell count (RBCs), hemoglobin level ( $\mathrm{Hb}$ ), hematocrit (packed cell volume), mean corpuscular volume (MCV), and platelet count, were analyzed with an automated hematology analyzer, DxH 800 (Beckman Coulter (UK) Ltd, High Wycombe, UK).

\section{Measurement of Left Ventricular Mass (LVM) and Ejection Fraction (EF)}

Left ventricular mass (LVM) was calculated according to the guidelines of the American Society of Echocardiography and the European Association of Cardiovascular Imaging, using linear measurements derived from 2-D images. ${ }^{18}$ LVM was indexed to body surface area (BSA) $\left(\mathrm{g} / \mathrm{m}^{2}\right)$ and the result referred to as the left ventricular mass index (LVMI). ${ }^{19}$ Ejection fraction (EF) was also assessed in study patients by $2 \mathrm{D}$ echocardiography using Modified Simpson method (biplane method of disks), which requires tracing the LV endocardial border in the apical 4- and 2-chamber views in both end-diastole and end-systole. ${ }^{20}$

\section{Outcome Assessments}

Electronic medical records of the patients were checked, and $\mathrm{ACM}$ was documented along with the corresponding diagnosis and date. Patients or their next of kin were contacted for other mortality incidents that might have occurred and documented in other hospitals. Deaths were considered cardiovascular unless a specific non-cardiovascular cause was identified. Cardiovascular deaths were classified according to the Candesartan in Heart failure: Assessment of Reduction in Mortality and Morbidity (CHARM) program. ${ }^{5}$

\section{Statistical Analysis}

Baseline data are reported as mean \pm SD for continuous variables and as number and percentages for categorical variables. Median and ranges of values were used for nonnormally distributed data. Comparison between patient groups was done using unpaired Student's $t$-test, MannWhitney $U$-test, and chi-square test, depending on the type of data. To assess the association between biochemical and clinical variables with ACM, univariate and multivariate Cox-proportional hazards models, with chi-squared statistic test to assess the relationship between all covariates in the model were used. The number of variables included in the multivariate analysis was based on a ratio of events per variable (EPV) of $10 .^{21}$ Time scale of the Cox model was 24 months from the date of the baseline of serum electrolyte or study entry. Kaplan-Meier survival analysis with Log-rank test was used to compare survival curves for subgroup analysis. All statistical analyses were performed using Minitab statistical software (version18, Minitab Inc., State College, PA, USA) and MedCalc statistical software (version 19.1.13, MedCalc software, Ostend, Belgium). A value of $p<0.05$ was considered statistically significant.

\section{Results \\ Baseline Characteristics}

A total of 241 patients were included in the final analysis, with a mean age of $60.61 \pm 12.63(95 \% \mathrm{CI} ; 59.01,62.22)$ years. $65.1 \%$ of the patients were males, and $75.5 \%$ of them were Saudi. The population sample was overweight-obese, along with a high prevalence of type 2 DM (70.9\%) (Table 1). The etiology of CHF was predominantly ischemic and approximately half of the patients had mild-to-moderate disease (NHYA class I-II). Other significant comorbid conditions included systemic hypertension (in $77.2 \%$ of the patients), and dyslipidaemia (in 56.0\%). The patients were on optimal anti-failure medication, as summarised in Table 1.

Measurement of patient biochemical parameters, as summarized in Table 2, showed values for serum sodium which fell within the normal lab range of 135-145 mEq/L. Likewise, serum potassium and chloride were within normal limits; however, patients' $\mathrm{CO}_{2}$ concentration and blood urea nitrogen were elevated (normal ranges; $23-29 \mathrm{mEq} / \mathrm{L}$ and $5-20$ $\mathrm{mg} / \mathrm{dL}$, respectively). Measurement of hematological parameters showed normal serum hemoglobin (normal range; 12-14 g/dL), as well as normal hematocrit and MCV levels. The main echocardiographic features, LVMI and EF, are listed in Table 2. 
Table I Baseline Clinical Characteristics of Study Patients as One Group and Classified Based on Serum Sodium Concentrations

\begin{tabular}{|c|c|c|c|c|}
\hline Characteristics & $\begin{array}{l}\text { All Patients } \\
\mathrm{N}=24 \text { I }\end{array}$ & $\begin{array}{l}\text { Patients with } \mathrm{Na} \geq 135 \\
\mathrm{~N}=207\end{array}$ & $\begin{array}{l}\text { Patients with } \mathrm{Na}<135 \\
\mathrm{~N}=34\end{array}$ & $P$ value \\
\hline Age (years) & $60.61 \pm 12.63$ & $60.6 \pm 12.7$ & $60.6 \pm 12.4$ & 0.978 \\
\hline Male sex & 157 (65.1\%) & 135 (65.2\%) & 22 (64.7\%) & 0.954 \\
\hline Height $(\mathrm{cm})^{*}$ & $162.47 \pm 9.74$ & $162.45 \pm 9.78$ & $162.59 \pm 9.64$ & 0.940 \\
\hline Weight (kg) & $76.00(67.00,89.00)$ & $76.00(66.00,90.00)$ & $77.50(69.85,83.00)$ & 0.912 \\
\hline BMI $\left(\mathrm{kg} / \mathrm{m}^{2}\right)^{*}$ & $29.00(25.00,33.30)$ & $28.95(24.58,33.70)$ & $29.35(25.30,31.60)$ & 0.792 \\
\hline Nationality (Saudi) & $182(75.52 \%)$ & $158(76.33 \%)$ & $24(70.59 \%)$ & 0.471 \\
\hline NYHA class** & & & & 0.459 \\
\hline 1 & 32 (13.73\%) & 27 (13.5\%) & $5(15.15 \%)$ & \\
\hline 2 & $90(38.63 \%)$ & 81 (40.5\%) & $9(27.27 \%)$ & \\
\hline 3 & 75 (32.19\%) & 61 (30.5\%) & $14(42.42 \%)$ & \\
\hline 4 & $36(15.45 \%)$ & $31(15.5 \%)$ & $5(15.15 \%)$ & \\
\hline \multicolumn{5}{|l|}{ Etiology of CHF } \\
\hline IHD/non-ischemic & $160(66.67 \%) / 80(33.33 \%)$ & $136(66.02 \%) / 70(33.98 \%)$ & $24(70.59 \%) / 10$ (29.4l\%) & 0.601 \\
\hline \multicolumn{5}{|l|}{ Co-morbidities } \\
\hline Hypertension & $186(77.18 \%)$ & $157(75.85 \%)$ & $29(85.29 \%)$ & 0.224 \\
\hline Type I diabetes mellitus & $\mathrm{I}(0.4 \mathrm{I} \%)$ & 0 & I (2.94\%) & - \\
\hline Type 2 diabetes mellitus & $171(70.95)$ & $142(68.60 \%)$ & $29(85.29 \%)$ & 0.047 \\
\hline Dyslipidaemia & $135(56.02 \%)$ & $114(55.07 \%)$ & $21(61.76 \%)$ & 0.466 \\
\hline Arrhythmias***/AF & $54(22.41 \%) / 48(19.92 \%)$ & $45(21.74 \%) / 40(19.32 \%)$ & 9 (26.47\%)/8(23.53\%) & $0.540 / 0.569$ \\
\hline Transient ischemic attacks & $3(1.24 \%)$ & $2(0.97 \%)$ & I (2.94\%) & - \\
\hline Stroke & 27 (II.20\%) & $24(11.59 \%)$ & $3(8.82 \%)$ & 0.635 \\
\hline COPD & $26(10.79 \%)$ & $19(9.18 \%)$ & 7 (20.59\%) & 0.047 \\
\hline Bronchial asthma & $22(9.13 \%)$ & $19(9.18 \%)$ & $3(8.82 \%)$ & 0.947 \\
\hline Chronic liver disease & $8(3.32 \%)$ & $8(3.86 \%)$ & 0 & 0.244 \\
\hline \multicolumn{5}{|l|}{ Cardiac medications and others } \\
\hline ACEls/ARBs & $135(56.02 \%) / 73(30.29 \%)$ & $117(56.52 \%) / 64(30.92 \%)$ & $18(52.94 \%) / 9(26.47 \%)$ & $0.697 / 0.601$ \\
\hline Beta blockers/CCBs & $199(82.57 \%) / 53(21.99 \%)$ & $17 \mid(82.61 \%) / 44(21.26 \%)$ & $28(82.35 \%) / 9(26.47 \%)$ & $0.971 / 0.496$ \\
\hline Aspirin/Clopidogrel & $199(82.57 \%) / 91$ (37.76\%) & $172(83.09 \%) / 74(35.75 \%)$ & $27(79.41 \%) / 17(50 \%)$ & $0.600 / 0.112$ \\
\hline Warfarin & $37(15.35 \%)$ & $33(15.94 \%)$ & $4(11.76 \%)$ & 0.531 \\
\hline Thiazide/Loop diuretics & $43(17.84 \%) / 160(66.39 \%)$ & $38(18.36 \%) / 133(64.25 \%)$ & $5(14.71 \%) / 27(79.41 \%)$ & $0.606 / 0.083$ \\
\hline Spironolactone & $89(36.93 \%)$ & $78(37.68 \%)$ & II (32.35\%) & 0.551 \\
\hline Digoxin & $36(14.94 \%)$ & $28(13.53 \%)$ & $8(23.53 \%)$ & 0.129 \\
\hline Nitrates & $68(28.22 \%)$ & $56(27.05 \%)$ & $12(35.29 \%)$ & 0.322 \\
\hline Statins & $174(72.20 \%)$ & $148(71.50 \%)$ & $26(76.47 \%)$ & 0.549 \\
\hline Insulin & $72(29.88 \%)$ & $59(28.50 \%)$ & $13(38.24 \%)$ & 0.251 \\
\hline Sulphonylureas & $54(22.41 \%)$ & $49(23.67 \%)$ & $5(14.71 \%)$ & 0.245 \\
\hline Metformin & $100(41.49 \%)$ & $84(40.58 \%)$ & $16(47.06 \%)$ & 0.477 \\
\hline Thiazolidinediones & $2(0.83 \%)$ & $2(0.97 \%)$ & - & - \\
\hline Dipeptidyl peptidase-4 inhibitors & $26(10.79 \%)$ & $21(10.14 \%)$ & $5(14.71 \%)$ & 0.427 \\
\hline
\end{tabular}

Notes: Values are expressed as mean \pm SD or number (percentage of patients). Median with interquartile ranges are used for non-normally distributed data. Missing data from medical records: *15 patients did not have their height, **8 patients did not have NYHA class, ***Other 6 arrhythmias (premature ventricular contractions, atrial flutter, 2 supraventricular tachycardia, and 2 ventricular tachycardia).

Abbreviations: BMI, body mass index; NYHA, New York Heart Association; IHD, ischemic heart disease; AF, atrial fibrillation (paroxysmal and permanent); COPD, chronic obstructive pulmonary disease; ACEls, angiotensin=converting enzyme inhibitors; ARBs, angiotensin receptor blockers; CCBs, calcium channel blockers.

\section{Study Population as per Serum Sodium Concentration}

Classifying the patients according to serum sodium levels falling below $135 \mathrm{mEq} / \mathrm{L}$ revealed that $14.1 \%$ of the population had hyponatremia. When matched for age, sex, BMI, severity, and etiology of CHF, patients with hyponatremia had a significantly higher percentage of type $2 \mathrm{DM}$ and COPD (Table 1). They had a trend for a higher percentage of systemic hypertension, higher use of clopidogrel, furosemide, digoxin, and insulin, but these did not reach statistical significance 
(Table 1). Patients with hyponatremia also had significantly lower chloride concentration, a smaller anion gap, and a higher WBC count (Table 2).

\section{Study Endpoints}

Within 24 months from the serum sodium measurements, 46 fatalities occurred, 41 of which were considered cardiovascular deaths. The causes of deaths and the number that died of each cause were as follows: 14 patients died of cardiorespiratory arrest complicating $\mathrm{CHF}, 7$ from coronary events, 5 from stroke, 5 from cardiogenic shock, 1 from acute pulmonary edema, 1 from coronary artery bypass grafting complications, 1 from constrictive pericarditis; 4 deaths with histories suggestive of cardiovascular death occurred out-of-hospital; and there were 3 deaths in the hospital with missing death certificates. The five other deaths were attributed to sepsis/septic shock.

Univariate analysis for ACM showed that age, NYHA class (III-IV vs I-II), BUN, eGFR, RBCs, Hb, hematocrit, LVMI, and type 2 DM had significant associations with ACM (Table 3). Sex, BMI, serum creatinine, sodium, potassium, chloride, $\mathrm{CO}_{2}, \mathrm{AG}, \mathrm{HbA} 1 \mathrm{c}$, and $\mathrm{EF}$ did not show any significant associations with ACM. Multivariate analysis for ACM included age, NYHA class (III-IV vs I-

Table 2 Biochemical, Haematological and Echocardiographic Characteristics of Study Patients as One Group and Classified Based on Serum Sodium Concentrations

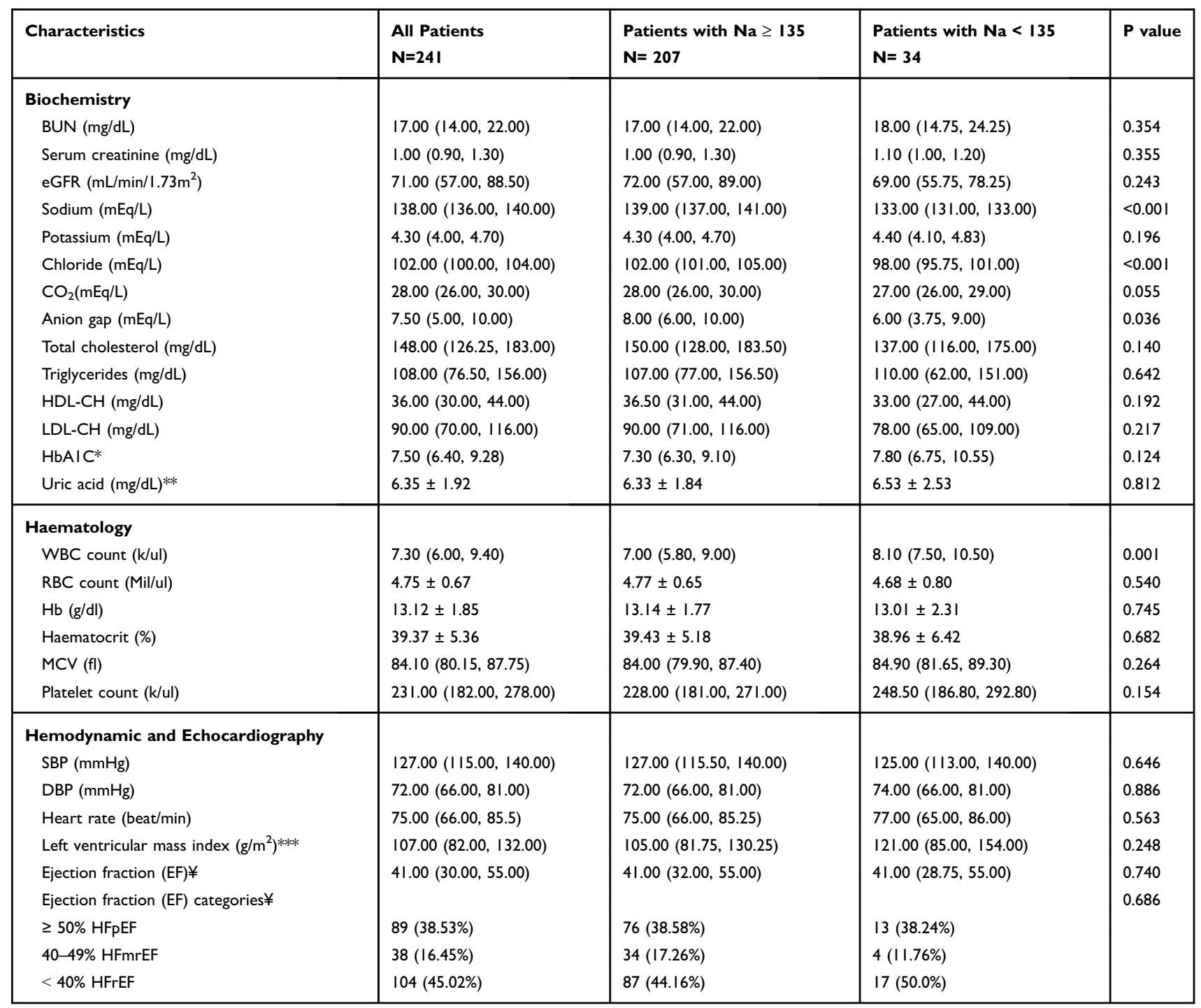

Notes: Values are expressed as mean \pm SD or number (percentage of patients). Median with interquartile ranges are used for non-normally distributed data. Missing data from medical records: *data based on 164 patients, ** data based on 82 patients, ***data based on 217 patients, $¥$ data from 231 patients.

Abbreviations: WBC, white blood cell; RBC, red blood cell; Hb, hemoglobin; MCV, mean corpuscular volume; BUN, blood urea nitrogen; eGFR, estimated glomerular filtration rate; HDL, high-density lipoprotein; $\mathrm{CH}$, cholesterol; LDL, low-density lipoprotein; HbAIC, glycated hemoglobin; SBP, systolic blood pressure; DBP, diastolic blood pressure; $\mathrm{HFpEF}$, heart failure with preserved EF; HFmrEF, heart failure with mid-range EF; HFrEF, heart failure with reduced EF. 
Table 3 Univariate and Multivariate Cox-Proportional Hazard Models for All-Cause Mortality in the Study Population

\begin{tabular}{|c|c|c|}
\hline Variable & HR $(95 \% \mathrm{Cl})$ & $P$-value \\
\hline \multicolumn{3}{|l|}{ Univariate Analysis } \\
\hline Age (years) & $1.07(1.04,1.10)$ & $<0.001$ \\
\hline NYHA class (III-IV vs I-II) & $3.57(1.74,7.32)$ & $<0.001$ \\
\hline BUN (mg/dL) & $1.03(1.01,1.06)$ & 0.016 \\
\hline $\mathrm{eGFR}\left(\mathrm{mL} / \mathrm{min} / 1.73 \mathrm{~m}^{2}\right)$ & $0.98(0.97,0.99)$ & 0.006 \\
\hline RBCs (Mil/ul) & $0.47(0.29,0.76)$ & 0.002 \\
\hline $\mathrm{Hb}(\mathrm{g} / \mathrm{dL})$ & $0.72(0.61,0.86)$ & $<0.001$ \\
\hline Hematocrit (\%) & $0.89(0.83,0.94)$ & $<0.001$ \\
\hline $\operatorname{LVMI}\left(g / \mathrm{m}^{2}\right)$ & $1.01(1.00,1.01)$ & 0.037 \\
\hline Type 2 DM (yes) & $3.69(1.46,9.34)$ & 0.006 \\
\hline Serum Sodium (mEq/L) & $0.96(0.88,1.04)$ & 0.342 \\
\hline Serum Sodium $(<135$ vs $\geq 135 \mathrm{mEq} / \mathrm{L})$ & $1.59(0.77,3.30)$ & 0.211 \\
\hline Serum Chloride (mEq/L) & $0.95(0.88,1.03)$ & 0.247 \\
\hline \multicolumn{3}{|l|}{ Multivariate analysis } \\
\hline Age (years) & $1.06(1.03,1.09)$ & $<0.001$ \\
\hline NYHA class (III-IV vs I-II) & $2.31(1.11,4.82)$ & 0.026 \\
\hline $\operatorname{eGFR}\left(\mathrm{mL} / \mathrm{min} / 1.73 \mathrm{~m}^{2}\right)$ & $0.99(0.97,1.00)$ & 0.096 \\
\hline LVMI $\left(g / \mathrm{m}^{2}\right)$ & $1.01(1.00,1.02)$ & 0.039 \\
\hline Type 2 DM (yes) & $3.03(1.13,8.16)$ & 0.028 \\
\hline
\end{tabular}

Notes: Overall Model Fit. Null model -2 Log Likelihood 495.515. Full model -2 Log Likelihood 424.79I. Chi-squared 70.724. DF 5. Significance level: $P<0.000$ I.

II), eGFR, LVMI, and type 2 DM showed that age, NYHA class (III-IV vs I-II), LVMI, and type 2 DM had significant associations with $\mathrm{ACM}$ in the study population, with HRs of; $1.06(\mathrm{P}<0.001), 2.31(\mathrm{P}=0.026), 1.01 \quad(\mathrm{P}=0.039)$, and $3.03(\mathrm{P}=0.028)$, respectively. Similar results were obtained when the model included hematocrit instead of eGFR (data not shown).

\section{Subgroup Analysis}

In an attempt to focus more on the significance of hyponatremia in patients with moderate-severe heart failure, and based on an interaction that was found between NYHA (III-IV vs I-II) with serum sodium $(\geq 135$ vs $<135$ $\mathrm{mEq} / \mathrm{L}$ ) (interaction plot not shown), the multivariate analysis for ACM was repeated with the same variables; however, NYHA class and serum sodium concentration were sub-classified into four groups, in an attempt to test the interaction between them. Group 1 (reference group); NYHA I-II with normal sodium concentration (108 patients), group 2; NYHA III-IV with normal sodium concentration (92 patients), group 3; NYHA I-II with hyponatremia (14 patients), and group 4; NYHA III-IV with hyponatremia (19 patients). The risk of ACM was; HR $2.10(95 \% \mathrm{CI} ; 0.95,4.64)(\mathrm{P}=0.067)$ in group 2 vs 1 ,
HR $0.93(95 \%$ CI; 0.11, 7.46) $(\mathrm{P}=0.942)$ in group 3 vs 1 , and HR $3.39(95 \% \mathrm{CI} ; 1.21,9.49)(\mathrm{P}=0.020)$ in group $4 \mathrm{vs}$ 1. The four groups survival curves were plotted and compared by Log-rank test and showed a significant difference in Figure $2(\mathrm{P}<0.001)$.

\section{Discussion}

We sought via this observational study to explore the association of hyponatremia with ACM in a population with a different genetic makeup and co-morbidities, and the results can be summarized by the following points. Firstly, the prevalence of hyponatremia in this CHF population (14\%) is broadly similar to some reports $(13.8 \% \text {, and } 15.2 \%)^{10,22}$ but lower than other reports in the literature (57\% - using a different cut-off value of $\leq 137,17 \%$, and $51 \%)^{8,9,11}$ The later observation may be due to the fact that two of the referenced studies included patients with more advanced CHF than ours. ${ }^{8,11}$ Secondly, significant predictors of ACM in the study population were: type $2 \mathrm{DM}$, NYHA (III-IV vs I-II), age, and LVMI in order of significance. However, the subgroup analysis revealed that hyponatremia can be a biomarker of poorer prognosis among patients with moderate-to-severe CHF. This observation is in agreement with some of the aforementioned studies that exclusively selected patients with ejection fraction $<30 \%{ }^{8}$ or NYHA class III-IV. ${ }^{11}$ Our results can be explained by the fact that disease severity correlates significantly with the plasma concentrations of certain neurohormones involved directly or indirectly in modulating serum sodium, including; norepinephrine, arginine vasopressin, atrial natriuretic peptide, and renin activity. ${ }^{23}$ Thirdly, hyponatremia in this study population might reflect a number of underlying mechanisms. Normal uric acid concentration and normal anion gap might suggest a relative volume depletion as a contributory factor. ${ }^{24}$ An observation of note potentially relating to this finding is the percentage of patients maintained on furosemide therapy - a well-known agent that predisposes to hyponatremia, ${ }^{25}$ and hypochloremia, ${ }^{26}$ interpreted cautiously in relation to the percentage of patients with moderate-to-severe CHF. Hyperglycemia could be another contributory factor in our cohort (as judged with suboptimal glycated hemoglobin). Finally, the association of hyponatremia with type $2 \mathrm{DM}$ is a significant finding and is well supported in the literature. It is worth noting that the diabetic state is associated with hyponatremia that is attributable to numerous underlying pathological mechanisms. Dilutional hyponatremia results from hyperglycemia due to osmotic diuresis. ${ }^{27,28}$ Pseudohyponatremia occurs with marked hypertriglyceridemia and hyperproteinemia. ${ }^{28}$ Moreover, drug-induced hyponatremia occurs in patients with DM and can be attributed to more 

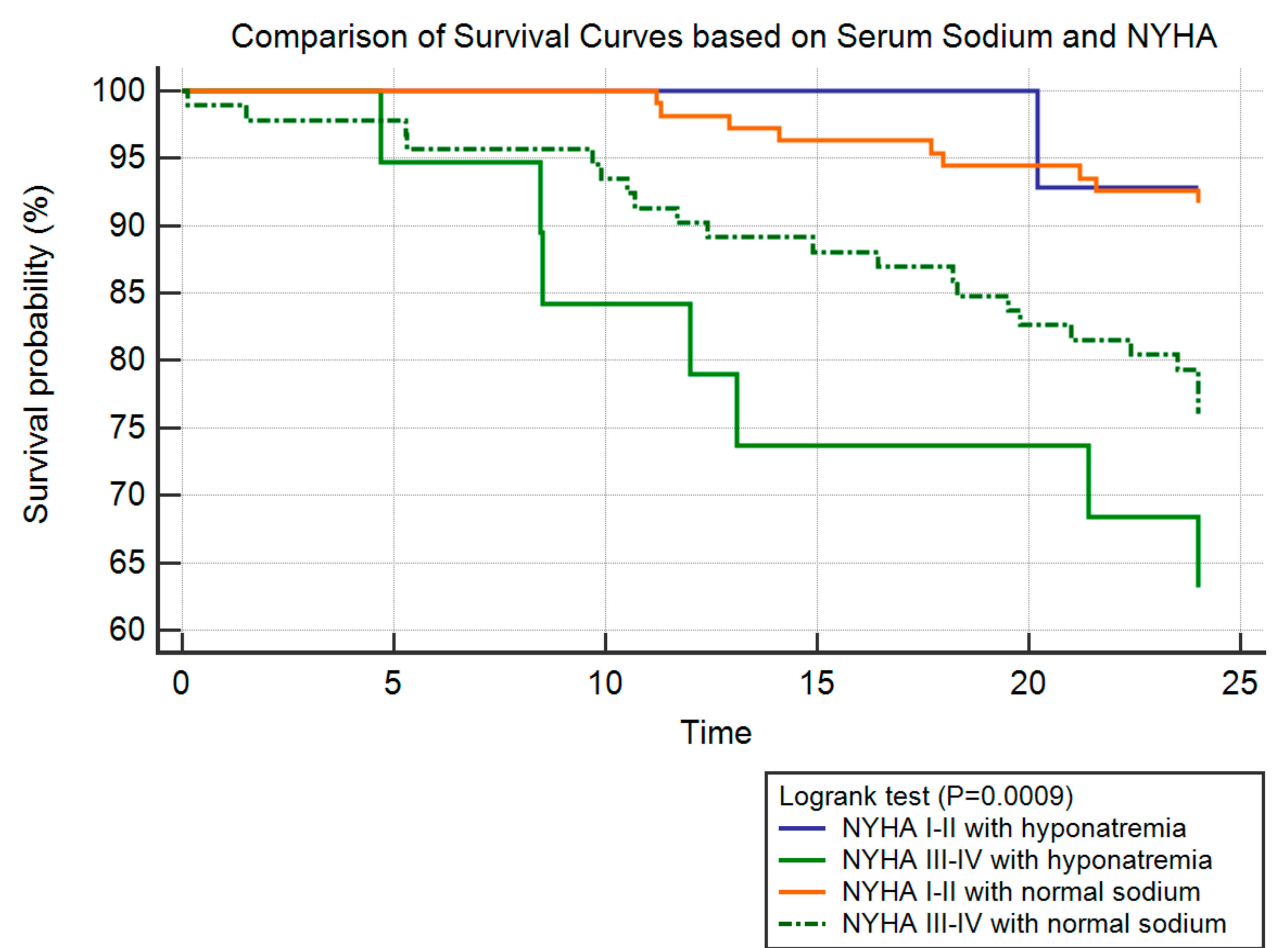

Figure 2 Kaplan-Meier survival analysis in CHF patients based on NYHA class, and serum sodium ( $\geq 135$, or $<135 \mathrm{mEq} / \mathrm{L})$.

than one pharmacological class. Insulin potentiates vasopressin-induced aquaporin 2 expression in renal collecting ducts. ${ }^{29}$ Metformin, via adenosine monophosphate kinase (AMPK) activation, stimulates the phosphorylation of aquaporin 2 and urea transporter A1 and potentiates the peripheral action of vasopressin. ${ }^{30,31}$ This is in addition to hyponatremia resulting from first-generation sulphonylureas that are rarely used nowadays, and diuretics that might be prescribed at later stages of diabetic nephropathy. DM associated hyponatremia is yet another mechanism that has been described and attributed to an elevated plasma vasopressin concentration, independent of hyperglycemia. ${ }^{32} \mathrm{DM}$ in our cohort was the strongest predictor of mortality, and this is consistent with other studies in the literature. ${ }^{4,33}$ However, a significantly higher prevalence of type $2 \mathrm{DM}$ in our population (71\%) was found in comparison to other CHF populations $(28 \%, 17.6 \%, 42 \%, 23.7 \%$, and $26.7 \%$ ). ${ }^{4,6,9,10,12}$ Such alarming prevalence, and negative prognostic value in CHF patients, along with BMI and fasting blood glucose as the top two risk factors in Saudi population, ${ }^{34}$ dictates more attention and efficient preventive strategies in our patient population.

In this study, we excluded patients with other confounding co-morbidities, such as hereditary anemia and nutritional anemia because of the effect of these conditions on survival and clinical outcomes in heart failure. ${ }^{35} \mathrm{We}$ also excluded patients with cancer/malignancy due to its direct influence on survival and the possibility of tumorassociated SIADH secretion. ${ }^{36}$ Patients with advanced kidney disease who were likely to have hyponatremia and other electrolyte imbalances were also excluded. ${ }^{37}$

\section{Limitations}

This study has a number of limitations. Proper assessment of hyponatremia involves the correction of serum sodium, as per blood glucose concentration. ${ }^{38}$ Such correction was not feasible as glycemic control is routinely assessed in our hospital by glycated hemoglobin, and not be random blood glucose concentration. Another limitation is the lack of other important biomarkers such as brain natriuretic peptide $^{39}$ (which is not measured routinely in our hospital) to allow for prognostic comparisons. The duration of CHF diagnosis is not documented in patient's electronic records, so it is unclear if this missing variable could have any prognostic implications. Finally, the subgroup analysis done resulted in a smaller number of patients per group, which would have resulted in underpowered results.

\section{Conclusion}

CHF is a growing public health problem worldwide, with significant morbidity and mortality; therefore, the search 
for simple and cost-effective serum biomarkers to guide proper management is warranted. Hyponatremia is a common electrolyte abnormality that has a prognostic value for clinical outcomes, including ACM. This study has shown that the prevalence of hyponatremia in this sample of Saudi patients with CHF is almost similar to, or less than that of other populations. ACM was independently predicted by type 2 DM, NYHA class, age, and LVMI. Despite that hyponatremia was not found to be an independent predictor of ACM in our population, it was associated with a reduced survival among patients with moderate-to-severe disease.

\section{List of Abbreviations}

CHF, Chronic Heart Failure; ACM, All-cause mortality; LVM, Left Ventricular Mass; EF, Ejection Fraction; IHD, Ischemic Heart Disease; LVMI, Left Ventricular Mass Index; SD, Standard Deviation; HRs, Hazards Ratios; HR, Hazards Ratio; NYHA, New York Heart Association; DM, Diabetes Mellitus; ICH, International Conference on Harmonization; BMI, Body Mass Index; AF, Atrial Fibrillation; TIA, Transient Ischemic Attack; COPD, Chronic Obstructive Pulmonary Disease; ACEI, Angiotensin-Converting Enzyme Inhibitors; ARB, Angiotensin Receptor Blockers; CCBs, Calcium Channel Blockers; GFR, Glomerular Filtration Rate; HbA1C, Hemoglobin A1C (Glycated Hemoglobin); CBC, Complete Blood Count; WBCs, White Blood Cell Count; RBCs, Red Blood Cell Count; Hb, Hemoglobin; MCV, Mean Corpuscular Volume; BSA, Body Surface Area; $\mathrm{CO}_{2}$, Carbon Dioxide; AG, Anion Gap; HFrEF, Heart Failure with Reduced Ejection Fraction; HFmrEF, Heart Failure with Mid-Range Ejection Fraction; BUN, Blood Urea Nitrogen; LDL, Low-Density Lipoprotein; CH, Cholesterol; HDL, High-Density Lipoprotein; eGFR, Estimated Glomerular Filtration Rate; SIADH, Syndrome of Inappropriate Antidiuretic Hormone Secretion; CHARM, Candesartan in Heart failure: Assessment of Reduction in Mortality and Morbidity; STROBE, Strengthening the Reporting of Observational Studies in Epidemiology; KFHU, King Fahd Hospital of the University.

\section{Availability of Supporting Data}

The data that support the findings of this study are available on request from the corresponding author (MM. Alem).

\section{Ethical Approval and Consent to Participate}

The study protocol was approved by the institutional review board (IRB-2020-306-Pharm), Deanship of Scientific Research, Imam Abdulrahman bin Faisal University, Dammam, Saudi Arabia. Verbal consents were obtained from the patients included or their next of kin by telephone conversation.

\section{Acknowledgment}

I thank Dr Muruj Alshehri, Dr Mohammed Alelaiw, Dr Ali Almaa, and Dr Abdullah Alshehri for their participation in the data collection.

\section{Funding}

This research has not been funded by any source.

\section{Disclosure}

The author declares no conflict of interest.

\section{References}

1. Roger VL. Epidemiology of heart failure. Circ Res. 2013;113 (6):646-659. doi:10.1161/CIRCRESAHA.113.300268

2. Skrzynia C, Berg JS, Willis MS, Jensen BC. Genetics and heart failure: a concise guide for the clinician. Curr Cardiol Rev. 2015;11 (1):10-17. doi:10.2174/1573403X09666131117170446

3. Taylor CJ, Ordonez-Mena JM, Roalfe AK, et al. Trends in survival after a diagnosis of heart failure in the United Kingdom 2000-2017: population based cohort study. BMJ. 2019;364:1223. doi:10.1136/bmj.1223

4. Pocock SJ, Wang D, Pfeffer MA, et al. Predictors of mortality and morbidity in patients with chronic heart failure. Eur Heart J. 2006;27 (1):65-75. doi:10.1093/eurheartj/ehi555

5. Solomon SD, Anavekar N, Skali H, et al. Influence of ejection fraction on cardiovascular outcomes in a broad spectrum of heart failure patients. Circulation. 2005;112(24):3738-3744. doi:10.1161/ CIRCULATIONAHA.105.561423

6. Ezekowitz JA, McAlister FA, Armstrong PW. Anemia is common in heart failure and is associated with poor outcomes: insights from a cohort of 12065 patients with new-onset heart failure. Circulation. 2003;107(2):223-225. doi:10.1161/01.CIR.0000052622.51963.FC

7. Adlbrecht C, Kommata S, Hulsmann M, et al. Chronic heart failure leads to an expanded plasma volume and pseudoanaemia, but does not lead to a reduction in the body's red cell volume. Eur Heart J. 2008;29(19):2343-2350. doi:10.1093/eurheartj/ehn359

8. Lee WH, Packer M. Prognostic importance of serum sodium concentration and its modification by converting-enzyme inhibition in patients with severe chronic heart failure. Circulation. 1986;73 (2):257-267. doi:10.1161/01.CIR.73.2.257

9. Balling L, Schou M, Videbaek L, Hildebrandt P, Wiggers H, Gustafsson F. Prevalence and prognostic significance of hyponatraemia in outpatients with chronic heart failure. Eur J Heart Fail. 2011;13(9):968-973. doi:10.1093/eurjhf/hfr086

10. Bavishi C, Ather S, Bambhroliya A, et al. Prognostic significance of hyponatremia among ambulatory patients with heart failure and preserved and reduced ejection fractions. Am J Cardiol. 2014;113 (11):1834-1838. doi:10.1016/j.amjcard.2014.03.017 
11. Abebe TB, Gebreyohannes EA, Tefera YG, et al. The prognosis of heart failure patients: does sodium level play a significant role? PLoS One. 2018;13(11):e0207242. doi:10.1371/journal.pone.0207242

12. Rusinaru D, Tribouilloy C, Berry C, et al. Relationship of serum sodium concentration to mortality in a wide spectrum of heart failure patients with preserved and with reduced ejection fraction: an individual patient data meta-analysis (dagger): meta-Analysis Global Group in Chronic heart failure (MAGGIC). Eur $J$ Heart Fail. 2012;14(10):1139-1146. doi:10.1093/eurjhf/hfs099

13. Arampatzis S, Funk GC, Leichtle AB, et al. Impact of diuretic therapy-associated electrolyte disorders present on admission to the emergency department: a cross-sectional analysis. BMC Med. 2013;11(1):83. doi:10.1186/1741-7015-11-83

14. Alhabeeb W, Elasfar A, AlBackr H, et al. Clinical characteristics, management and outcomes of patients with chronic heart failure: results from the heart function assessment registry trial in Saudi Arabia (HEARTS-chronic). Int $J$ Cardiol. 2017;235::94-99. doi:10.1016/j.ijcard.2017.02.087

15. Yancy CW, Jessup M, Bozkurt B, et al. 2013 ACCF/AHA guideline for the management of heart failure: a report of the American college of cardiology foundation/American heart association task force on practice guidelines. J Am Coll Cardiol. 2013;62(16):e147-e239. doi:10.1016/j.jacc.2013.05.019

16. Vandenbroucke JP, Von EE, Altman DG, et al. Strengthening the Reporting of Observational Studies in Epidemiology (STROBE): explanation and elaboration. PLoS Med. 2007;4(10):e297. doi:10.1371/journal.pmed.0040297

17. Levey AS, Bosch JP, Lewis JB, Greene T, Rogers N, Roth D. A more accurate method to estimate glomerular filtration rate from serum creatinine: a new prediction equation. Modification of diet in renal disease study group. Ann Intern Med. 1999;130(6):461-470. doi:10.7326/0003-4819-130-6-199903160-00002

18. Lang RM, Badano LP, Mor-Avi V, et al. Recommendations for cardiac chamber quantification by echocardiography in adults: an update from the American Society of Echocardiography and the European Association of Cardiovascular Imaging. $J$ Am Soc Echocardiogr. 2015;28(1):1-39. doi:10.1016/j.echo.2014.10.003

19. Devereux RB, Lutas EM, Casale PN, et al. Standardization of M-mode echocardiographic left ventricular anatomic measurements. J Am Coll Cardiol. 1984;4(6):1222-1230. doi:10.1016/S0735-1097 (84)80141-2

20. Lang RM, Bierig M, Devereux RB, et al. Recommendations for chamber quantification: a report from the American Society of echocardiography's guidelines and standards committee and the chamber quantification writing group, developed in conjunction with the European association of echocardiography, a branch of the European society of cardiology. J Am Soc Echocardiogr. 2005;18 (12):1440-1463. doi:10.1016/j.echo.2005.10.005

21. Van DR, Hoeks S, Kardys I, Lenzen M, Boersma E. Tools and techniques-statistics: how many variables are allowed in the logistic and Cox regression models? EuroIntervention. 2014;9(12):14721473. doi:10.4244/EIJV9I12A245

22. Formiga F, Chivite D, Brase A, et al. Clinical characteristics and prognosis in patients with a first acute heart failure hospitalization according to admission hyponatremia. Acta Clin Belg. 2018;73 (4):281-286. doi:10.1080/17843286.2018.1429345
23. Benedict CR, Johnstone DE, Weiner DH, et al. Relation of neurohumoral activation to clinical variables and degree of ventricular dysfunction: a report from the registry of studies of left ventricular dysfunction. SOLVD Investigators. J Am Coll Cardiol. 1994;23 (6):1410-1420. doi:10.1016/0735-1097(94)90385-9

24. Decaux G, Schlesser M, Coffernils M, et al. Uric acid, anion gap and urea concentration in the diagnostic approach to hyponatremia. Clin Nephrol. 1994;42(2):102-108.

25. Liamis G, Milionis H, Elisaf M. A review of drug-induced hyponatremia. Am J Kidney Dis. 2008;52(1):144-153. doi:10.1053/j.ajkd.2008.03.004

26. Greenberg A. Diuretic complications. Am J Med Sci. 2000;319 (1):10-24. doi:10.1097/00000441-200001000-00002

27. Liamis G, Liberopoulos E, Barkas F, Elisaf M. Diabetes mellitus and electrolyte disorders. World J Clin Cases. 2014;2(10):488-496. doi:10.12998/wjcc.v2.i10.488

28. Turchin A, Seifter JL, Seely EW. Clinical problem-solving.. Mind the Gap $N$ Engl J Med. 2003;349(15):1465-1469. doi:10.1056/ NEJMcps031078

29. Bustamante M, Hasler U, Kotova O, et al. Insulin potentiates AVPinduced AQP2 expression in cultured renal collecting duct principal cells. Am J Physiol Renal Physiol. 2005;288(2):F334-F344. doi:10.1152/ajprenal.00180.2004

30. Efe O, Klein JD, LaRocque LM, Ren H, Sands JM. Metformin improves urine concentration in rodents with nephrogenic diabetes insipidus. JCI Insight. 2016;1(11). doi:10.1172/jci.insight.88409

31. Klein JD, Wang Y, Blount MA, et al. Metformin, an AMPK activator, stimulates the phosphorylation of aquaporin 2 and urea transporter A1 in inner medullary collecting ducts. Am J Physiol Renal Physiol. 2016;310(10):F1008-F1012. doi:10.1152/ajprenal.00102.2016

32. Bankir L, Bardoux P, Ahloulay M. Vasopressin and diabetes mellitus. Nephron. 2001;87(1):8-18. doi:10.1159/000045879

33. Barlera S, Tavazzi L, Franzosi MG, et al. Predictors of mortality in 6975 patients with chronic heart failure in the Gruppo Italiano per lo Studio della Streptochinasi nell'Infarto Miocardico-Heart Failure trial: proposal for a nomogram. Circ Heart Fail. 2013;6(1):31-39. doi:10.1161/CIRCHEARTFAILURE.112.967828

34. Tyrovolas S, El Bcheraoui C, Alghnam SA. The burden of disease in Saudi Arabia 1990-2017: results from the global burden of disease study 2017. Lancet Planet Health. 2020;4(5):e195-e208. doi:10.1016/S2542-5196(20)30075-9

35. Caramelo C, Justo S, Gil P. [Anemia in heart failure: pathophysiology, pathogenesis, treatment, and incognitae]. Rev Esp Cardiol. 2007;60(8):848-860. Spanish. doi:10.1157/13108999

36. Sorensen JB, Andersen MK, Hansen HH. Syndrome of inappropriate secretion of antidiuretic hormone (SIADH) in malignant disease. $J$ Intern Med. 1995;238(2):97-110. doi:10.1111/j.1365-2796.1995. tb00907.x

37. Sun L, Hou Y, Xiao Q, Du Y. Association of serum sodium and risk of all-cause mortality in patients with chronic kidney disease: A meta-analysis and systematic review. Sci Rep. 2017;7(1):15949. doi:10.1038/s41598-017-16242-3

38. Milani GP, Bianchetti MG, Lava SA. Measurement of sodium in heart failure. Heart. 2018;104(20):1724. doi:10.1136/heartjnl-2017312799

39. Brunner-la Rocca HP, Sanders-van WS. Natriuretic peptides in chronic heart failure. Card Fail Rev. 2019;5(1):44-49. doi:10.15420/cfr.2018.26.1 


\section{Publish your work in this journal}

The International Journal of General Medicine is an international, peer-reviewed open-access journal that focuses on general and internal medicine, pathogenesis, epidemiology, diagnosis, monitoring and treatment protocols. The journal is characterized by the rapid reporting of reviews, original research and clinical studies across all disease areas. The manuscript management system is completely online and includes a very quick and fair peer-review system, which is all easy to use. Visit http://www.dovepress.com/ testimonials.php to read real quotes from published authors. 